\title{
Evaluation of the success of sciatic nerve blockade by skin temperature measurement through infrared thermography
}

\author{
by F.G.A.M. van Haren ${ }^{\star \star \star \star}$, L. Kadic*, J.J. Driessen* (Arial, 10 pt, align right)
}

* Radboud University Medical Centre, PO Box 9101,6500HB, Nijmegen, The Netherlands.

** frank.vanharen@anes.radboudumc.nl.

\begin{abstract}
We hypothesized that blocking the sciatic nerve with ropivacaine (local anesthetic) will lead to a (measurable) temperature increase in innervated skin, and that this will correlate with existing sensory block tests. Therefore, 18 individuals undergoing foot surgery were studied. Skin temperature was measured just before nerve blockade and every 10 minutes thereafter.

Baseline skin temperatures showed a significant proximal to distal gradient. Thereafter, temperatures on increased significantly in the toes and foot. Pinprick correlates well to skin temperature, with an AUC in a Receiver Operating Curve of $85.9 \%(p<.001)$. This makes infrared skin temperature measuring a valuable test in determining block success.
\end{abstract}

\section{Introduction}

Neuraxial and peripheral nerve blocks are widely used for per-and postoperative pain relief in anaesthesia. Existing ways of testing the effectiveness of the block (pinprick, cold test, etc) are rather subjective, might not relate to the reaction to a surgical stimulus, and can be time-consuming or impossible to use (e.g. children or patients under anesthesia). [1]

Peripheral nerves contain sympathetic fibers. Their blockade by local anesthetic agents leads to vasodilatation and increased blood flow, and increases skin temperatures. [2]

Some studies have investigated the possibility of infrared thermography as a means to determine the success or failure of a peripheral nerve block, focusing mainly on brachial plexus (arm) blocks. This seems only partially possible. $[3,4,5,6]$ Research concerning the lower extremity is scarcer. $[7,8]$

In the present study we observed the relationship between ultrasound guided subgluteal sciatic nerve blocks and skin temperature by infrared thermography in the lower extremity. We hypothesized that blocking the sciatic nerve will lead to an increase in temperature of the skin area it innervates and that this will correlate to measurements with existing sensory tests.

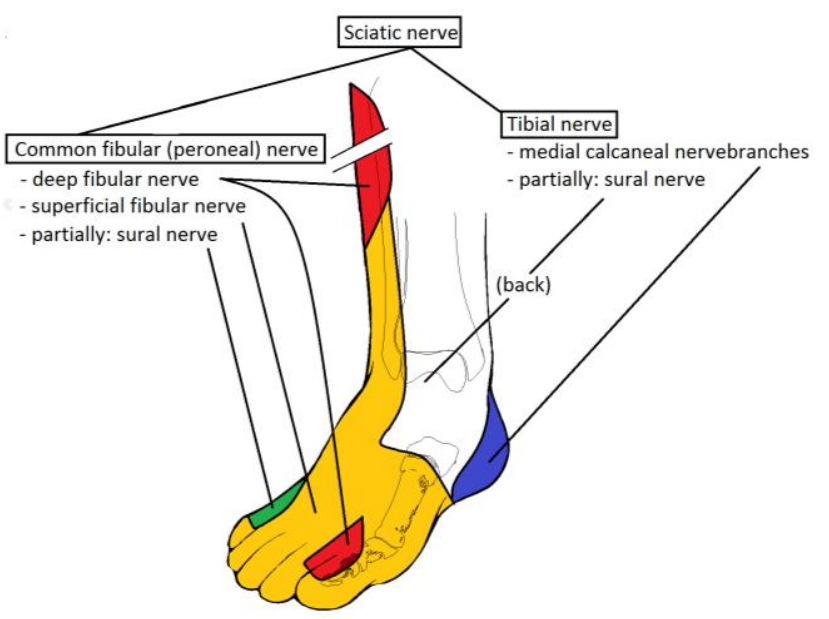

Figure 1. Skin innervation of the sciatic nerve and its branches.

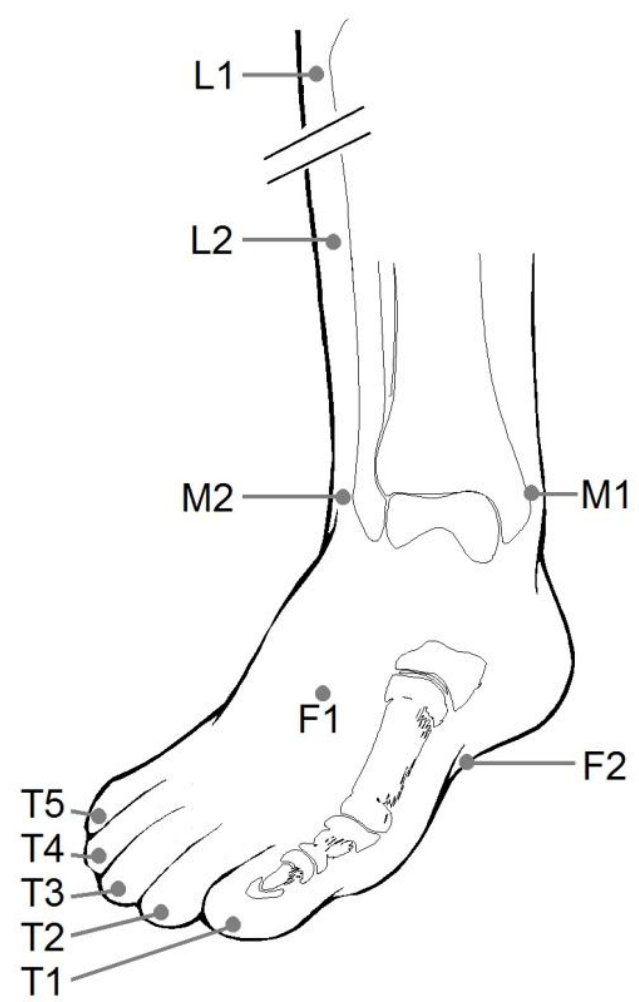

Figure 2. Measuring points visualized (T1-T5 = toe 1 through $5 ; F 1=$ foot dorsal; F2 = foot plantar; $M 1=$ medial malleolus; $M 2$ = lateral malleolus; $L 2=$ lower leg halfway knee and ankle; $L 1$ = lower leg at the knee). 


\section{Methods}

After approval by the regional research ethics committee, we studied 18 healthy individuals undergoing orthopaedic surgery of the ankle or foot under ultrasound-guided subgluteal blockade of the sciatic nerve with $30 \mathrm{ml}$ ropivacaine $7.5 \mathrm{mg} \cdot \mathrm{ml}-1$.

Skin temperature was measured on the toes, the dorsal and plantar side of the foot, the malleoli, and the lateral side of the lower leg, just before sciatic nerve blockade and at 10 minutes intervals thereafter (figure 1).

At each point in time, 3 infrared pictures were taken from the lateral side of the foot, the lower leg, and the medial and bottom side of the foot, of both legs. Except for pinprick testing, no interventions were performed during infrared imaging, and the patients were instructed not to move their legs during the measurement period (ThermaCAM ${ }^{\circledR}$ S65 Infrared Camera, FLIR Systems, Wilsonville, US). Camera settings were appropriate for room temperature, air humidity, emission coefficient for human skin (0.98), and a set distance of $0.8 \mathrm{~m}$.

Sensory assessment was performed by systematic pinprick testing with a $24 \mathrm{G}$ needle, consecutively from the lateral side of the lower leg (below the knee) over the lateral malleolus to the heel (1), the dorsal and lateral side of the foot (2), every toe separately (3), the plantar side of the foot (4). Results where noted as 'sharp' = 'normal' = 'positive' or 'numb' = 'negative'. These measuring sites were in accordance with the landmarks used for infrared measurement as seen in figure 2.

A general interpretation of the distribution of skin temperature took place on site. Afterwards, the absolute measurements were done with computer software (FLIR Systems, Wilsonville, US) at specific points within a measurement area of $10 \times 10$ pixels (about $2 \mathrm{~mm} 2$ of skin): 5 toes (T1, T2, T3, T4, T5), the dorsal (F1) and plantar (F2) side of the foot, the medial malleolus (M1) and lateral malleolus (M2) and the lateral side of the lower leg at the knee (L1) and halfway the lower leg (L2).

Temperatures $(T)$ were recorded with an accuracy of $+/-0.1$ degrees Celsius. Date are presented as 'mean $(95 \%$ confidence interval), with p-values $<0.05$ considered significant. For differences, a paired samples Students' t-test was used, to relate pinprick to continuous temperatures and determine the optimal cut-off point a Receiver Operating Characteristic (ROC) was used.

\section{Results}

18 patients were included (mean age 55.1 years, mean BMI $24.6 \mathrm{~kg} / \mathrm{m} 2$ ). No adverse events, signs of systemic toxicity of the local anaesthetic or post operative neurologic problems were seen.

Baseline skin temperatures showed a significant distal to proximal gradient. The temperature of the toes was lowest at $27.21^{\circ} \mathrm{C}\left(20.39-34.03^{\circ} \mathrm{C}\right)$ for T1-T2-T3-T4-T5 and the plantar side of the foot (F2) at $27.47^{\circ} \mathrm{C}\left(22.95-31.99^{\circ} \mathrm{C}\right)$, rising towards the dorsal side of the foot (F1) to $29.39^{\circ} \mathrm{C}(24.48-34.30)$ and medial and lateral malleolus (M1-M2) to $29.38^{\circ} \mathrm{C}\left(25.50-33.27^{\circ} \mathrm{C}\right)$, and the lower leg (L1-L2) at $30.12^{\circ} \mathrm{C}(27.58$ $\left.32.67^{\circ} \mathrm{C}\right)$.

After sciatic block, temperatures on the blocked side increased significantly in the toes and foot, but not in the medial malleolus and the lower leg (figure 3, figure 4).

When comparing pinprick to skin temperature in a Receiver Operating Curve (ROC), there was an AUC of $85.9 \%(95 \% \mathrm{Cl}=83.7-88.2 \%, p<0.001)$. There is an optimal sensitivity and specificity of $85.5 \%$ and $79.8 \%$ respectively (positive and negative predictive value of $77.5 \%$ and $87.1 \%$ respectively) at a temperature rise of $0.65^{\circ} \mathrm{C}$ from baseline values. The medial malleolus (not being innervated by the sciatic nerve) showed no significant difference to the lateral. 
http://dx.doi.org/10.21611/qirt.2014.022

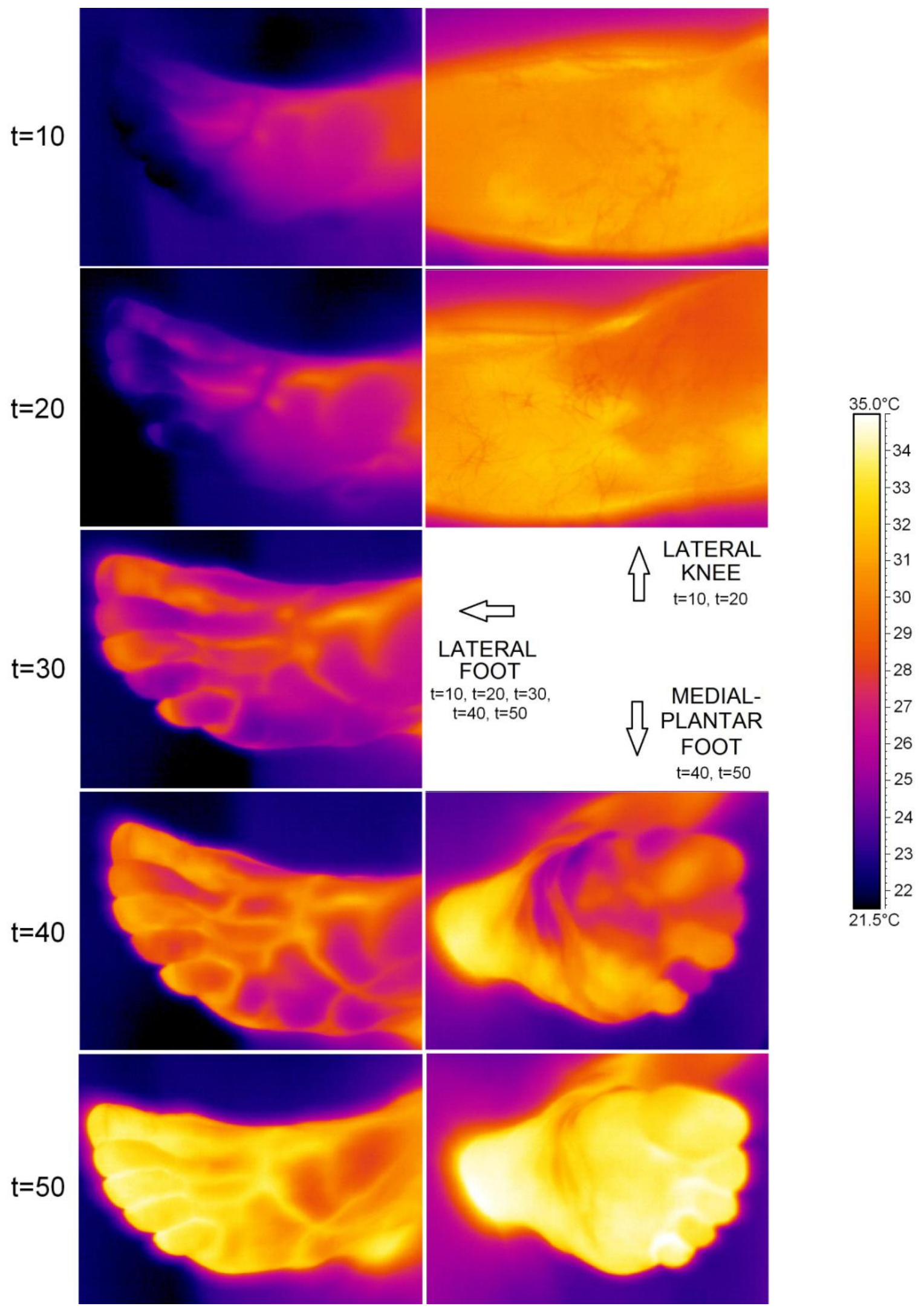

Figure 3. Example of infrared images at consecutive times. Shown are typical infrared pictures at 10 minute intervals after sciatic block. Notice the temperature rise starting in the toes and spreading cranially, the already higher baseline temperatures at the knee (not increasing further) at $t=10$ and $t=20$, and the similar temperatures for the plantar side of the foot at $t=40$ and $t=50$. The bar on the right shows the relationship between colors and specific temperatures. 


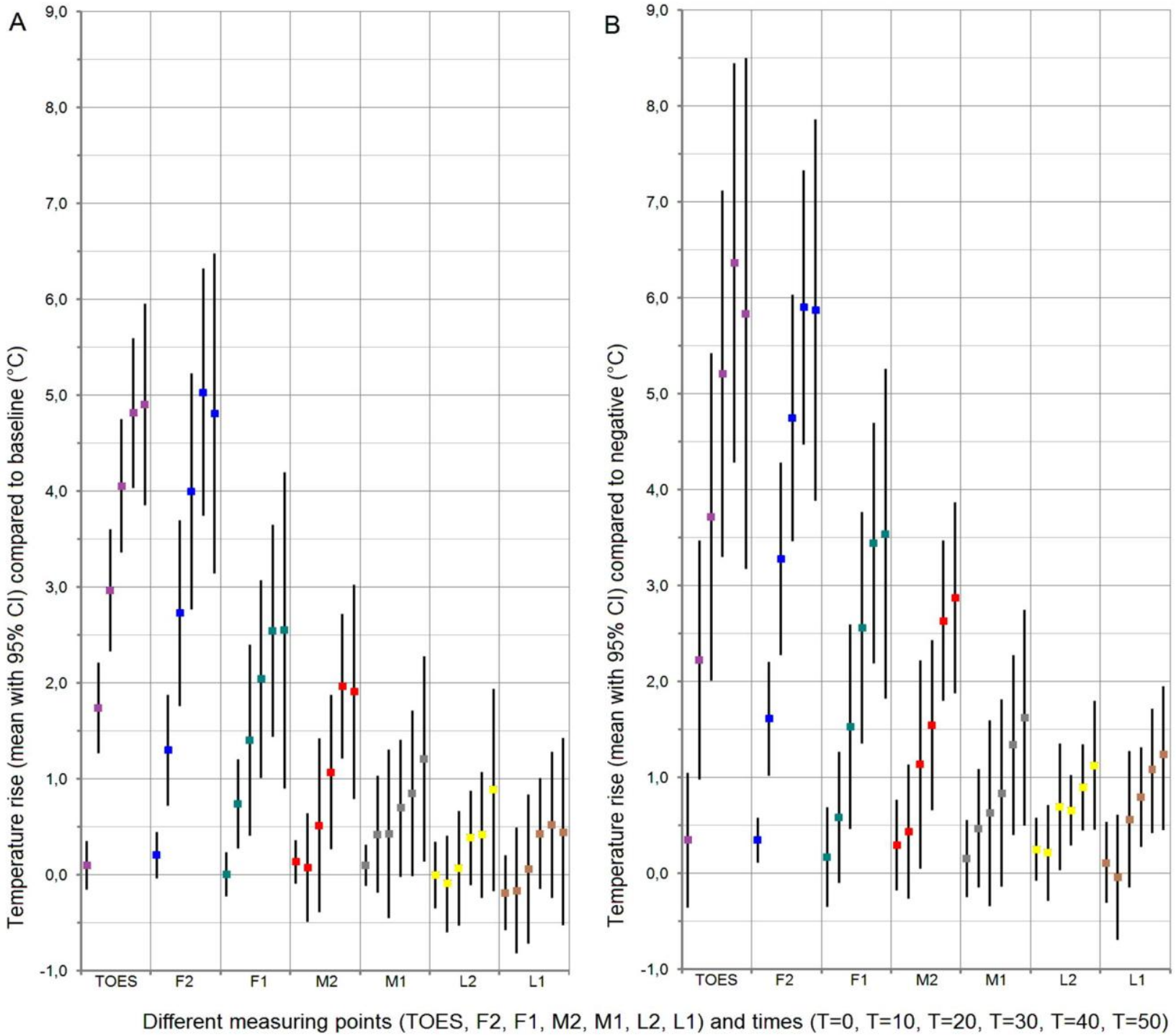

Figure 4. relationship between pinprick testing (positive and negative) and temperature change compared to baseline (cut-off $0.00^{\circ} \mathrm{C}$ on the left, cut-off $0.65^{\circ} \mathrm{C}$ on the right) for all measurements at specific times ( $n=184$ for $t=0 / 10 / 20 / 30 / 40$ and $n=173$ for $t=50)$ and for all measurements at all times $(n=1093)$. 


\section{Discussion}

After a sciatic nerve block, there was a higher and faster temperature rise in more distal areas of the skin. This could be explained by differences in baseline skin temperatures from core temperature. More proximal skin temperatures, as those on the lateral side of the knee, do not change significantly. The same phenomenon can be seen with neuraxial blocks, were almost no temperature rise occurs on the abdomen and proximal parts of the legs. [9]

In all cases, temperature rise precedes a negative pinprick, in slow as well as in fast arising blockades. This seems in accordance with the differences in speed of blockade of nerve fibers involved in this process, the fast blockade being in sympathetic C-fibers (with consequent temperature rise) compared to the slower blockade of the A-ס-fibers (with consequent fading of sensitivity for temperature and pain).

An earlier study by Lange et al on nerve blocks of the arm, showed that a temperature increase can easily be visualized after blocking the ulnar and median nerve, but not after blocking the musculocutaneous and radial nerve. Moreover, the areas of temperature rise and cutaneous innervations don't overlap. [3] Other research showed that the temperature rise in the limb is general when performing an axillary plexus block. [4] In studies by Werdehausen et al and Stevens et al the rise in skin temperature after epidural, spinal and combined sciatic and femoral blocks showed an evident proximal to distal gradient. $[7,8]$ However, because a whole limb was blocked, no definite statement could be made on the difference in temperature effects of the sciatic and femoral nerve.

In blocking the sciatic nerve, skin temperature rises bound to the area of innervations of the blocked nerve seem easily to be masked by the evident distal to proximal skin temperature gradient. Possibly, with including more patients, these differences could become significant. Furthermore, measuring skin temperatures after femoral blocks, or even after separate peroneal and tibial blocks, would provide more insight on the exact origination and existence of a differentiated temperature rise.

\section{Conclusion}

After sciatic nerve block, temperatures of the foot increased significantly. There was a good correlation between pinprick testing and infrared temperature measurement. This makes infrared skin temperature measuring a good and valuable test in determining block success when sensory testing is impossible as in small children or sleeping patients. 


\section{REFERENCES}

[1] Curatolo M, Petersen-Felix S, Arendt-Nielsen L. Assessment of regional analgesia in clinical practice and research. Br Med Bull 2005; 71: 61-76.

[2] Galvin EM, Niehof S, Verbrugge S, Maissan I, Jahn A, Klein J, van Bommel J. Peripheral flow index is a reliable and early indicator of regional block success. Anesth Analg 2006; 103(1): 239-243.

[3] Lange KHW, Jansen T, Ashgar S, Kristensen PL, Skjønnemand M, Nørgaard P. Skin temperature measured by infrared thermography after specific ultrasound-guided blocking of the musculocutaneous, radial, ulnar, and median nerves in the upper extremity. Br J Anaesth 2011; 106(6): 887-95.

[4] Galvin EM, Niehof S, Medina HJ, Zijlstra FJ, van Bommel J, Klein J, Verbrugge SJ. Thermographic temperature measurement compared with pinprick and cold sensation in predicting the effectiveness of regional blocks. Anesth Analg 2006; 102(2): 598-604.

[5] Hermanns H, Braun S, Werdehausen R, Werner A, Lipfert P, Stevens MF. Skin temperature after interscalene brachial plexus blockade. Reg Anesth Pain Med 2007; 32: 481-7.

[6] Minville V, Gendre A, Hirsch J, Silva S, Bourdet B, Barbero C, Fourcade O, Samii K, Bouaziz H. The efficacy of skin temperature for block assessment after infraclavicular brachial plexus block. Anesth Analg 2009; 108(3): 1034-6.

[7] Werdehausen R, Braun S, Hermanns H, Freynhagen R, Lipfert P, Stevens MF. Uniform Distribution of Skin-Temperature Increase After Different Regional-Anaesthesia Techniques of the Lower Extremity. Reg Anesth Pain Med 2007; 32: 73-78.

[8] Stevens MF, Werdehausen R, Hermanns H, Lipfert P. Skin temperature during regional anesthesia of the lower extremity. Anesth Analg 2006; 102: 1247-51.

[9] Van Haren FGAM, Driessen JJ, Kadic L, van Egmond J, Booij LHDJ, Scheffer GJ. The relation between skin temperature increase and sensory block height in spinal anaesthesia using infrared thermography. Acta Anaesth Scand 2010; 54(9): 1105-1110.Martin H., "Heat and Mass Transfer between Impinging Gas Jets and Solid Surfaces". Advances in Heat Transfer, vol. 13, pp. 1-60, Academic Press, New York, 1977. 